\title{
MIGRATION-RELATED PROTEIN ACTIVITY IN CELL ELECTROTAXIS***
}

\author{
ZBigniew Baster ${ }^{\mathrm{a}}$, Seawomir Lasota ${ }^{\mathrm{b}}$, Tomasz Witko ${ }^{\mathrm{a}}$ \\ Eliza ZimoląG ${ }^{\mathrm{b}}$, Jolanta Sroka ${ }^{\mathrm{b}}$, Zigigniew Madeja ${ }^{\mathrm{b}}$ \\ ZENON RAJFUR ${ }^{\text {a }}$
}

aThe M. Smoluchowski Institute of Physics, Faculty of Physics, Astronomy and Applied Computer Science, Jagiellonian University

Łojasiewicza 11, 30-348, Kraków, Poland

${ }^{\mathrm{b}}$ Department of Cell Biology, Faculty of Biochemistry, Biophysics and Biotechnology, Jagiellonian University

Gronostajowa 7, 30-387, Kraków, Poland

(Received August 17, 2017)

In this paper, we present an elegant and low-invasive method for bioimaging proteins activity on the molecular level in living cells. Using specially designed genetically encoded CFP-YFP FRET biosensors, proteins activation can be tracked by investigating probes FRET efficiency factor. This system allows to investigate cell physiology on the molecular level in studies of cell migration response to different stimuli, such as electric field.

DOI:10.5506/APhysPolB.48.1727

\section{Introduction}

Cell migration is a key process in development and functioning of singleand multi-cellular organisms. Active movement of cells underlies many physiological processes such as: embryological development, immune response, wound healing; and pathophysiological as metastasis [1]. In 2-dimensional movement, a cell usually migrates by creating a lamellipodium - a wide, flat projection made by F-actin filaments polymerization, which are continuously rearranged during cell movement process [2].

Small G-proteins from Rho family (Rho GTPases) are considered to be the cytoskeleton dynamics regulating factors, influencing cell polarization, migration, adhesion and proliferation processes [3, 4]. Our research concentrates on two proteins of the family: RhoA and Rac1. The former regulates

\footnotetext{
* Presented at the $2^{\text {nd }}$ Jagiellonian Symposium on Fundamental and Applied Subatomic Physics, Kraków, Poland, June 3-11, 2017.

** Corresponding authors: z.madeja@uj.edu.pl and zenon.rajfur@uj.edu.pl
} 
creation of stress fibers at the cell tail section, which contraction leads to its detachment from the substrate and displacement of the whole cell towards direction of the movement [5]. Rac1 activity is connected to actin polymerization at the leading edge of the cell. This way, it regulates a creation of lamellipodium $[2,3]$.

Many present biophysical studies concentrate on time and spatial correlation of protein activity with their physiological outcome. One of the very few experimental approaches is an application of FRET biosensors, which together with fluorescence microscopy can indicate selected proteins activity with high resolution limited only by optical diffraction limit [6].

\section{FRET phenomenon}

The phenomenon of a nonradiative resonance energy transfer between two chromophores was first described in 1948 by Förster [7]. FRET is based on a long range dipole-dipole coupling between a Donor (D) and an Acceptor (A) molecules. For the process to occur, there are few requirements that have to be met:

- the donor emission spectrum and the acceptor absorption spectrum have to at least partially overlap [8];

- the donor emission dipole moment and the acceptor absorption dipole moment have to be in a similar spatial orientation [9];

- the distance between D-A has typically to be shorter than $100 \AA$ [10].

For FRET-related experiments, the main measured parameter is energy transfer efficiency $E$. It is connected to all of the requirements mentioned above, but usually most studies are based on its relationship to the distance between $\mathrm{D}-\mathrm{A}$ pair

$$
E(r)=\frac{R_{0}^{6}}{R_{0}^{6}+r^{6}},
$$

where $R_{0}$ is the Förster distance for which energy transfer efficiency is equal to $50 \%$, and $r$ is an actual distance between the donor and the acceptor [10].

\section{Single-chain FRET biosensors}

Currently, fluorescence biosensors are the only way to monitor spatial and time resolved protein activity in living cells. A protein whose activity is observed has to have at least two different spatial conformations dependent on its activation. Single-chain FRET biosensors are composed of two fluorescent proteins (FP) forming a FRET pair connected to the studied protein. The most common FRET pair derives from Cyan and Yellow Fluorescent Protein (CFP and YFP) families. A proper attachment of FPs to the 
protein allows observation of its activity by measuring the change of FRET efficiency factor caused by the protein conformational change. The example of FRET biosensors, that are used in our research is shown in Fig. 1 [11].

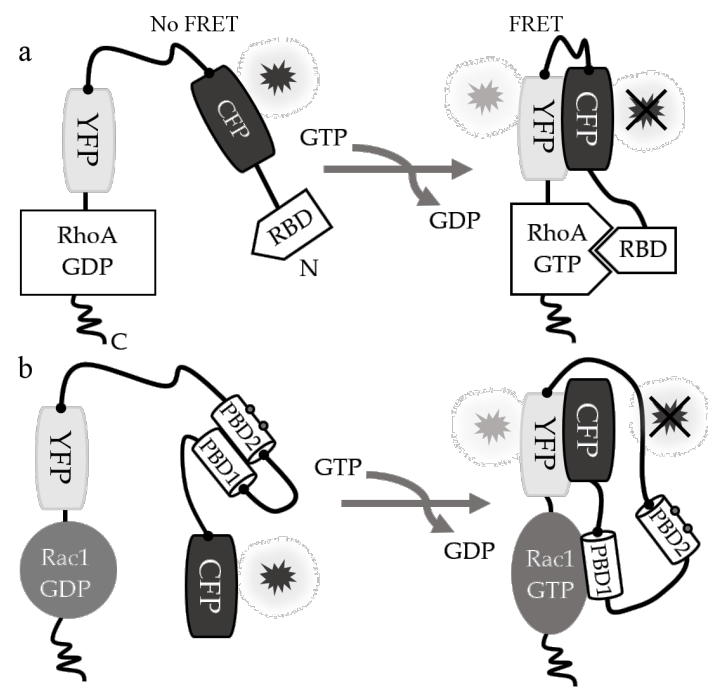

Fig. 1. Schemes of RhoA (a) and Rac1 (b) FRET biosensors. A GDP $\rightarrow$ GTP nucleotide exchange activates protein biological function. This also allows to bind Rho Binding Domain (RBD) or p21-Binding Domains (PBD), respectively, changing conformation of the whole biosensor. Redrawn from [12] and [13].

\section{Electrotactic response}

As mentioned earlier, cell migration is an absolutely indispensable component of many physiological processes. In order to be effective, it needs to be directed by chemical or physical factors. A well-known example of directional cell migration is chemotaxis, equally important is electrotaxis cell migratory response to direct current electric field (dcEF) [14]. In most cases, migration is directed to cathode, but some cell types have tendency to move towards anode [15]. Long-term studies showed that most cells exhibit response to physiological electric fields naturally formed during wound healing, embryonic development, neurogenesis and angiogenesis processes. Such reactivity is typical for many types of cancer cells as well, what suggests involvement of electrotaxis in cancer infiltration and metastasis [16].

Despite many years of research, complete mechanism underpinning response to dcEF has not been discovered. There are plenty of potential mechanisms proposed, mostly involving lateral migration of charged membrane proteins or alternatively voltage gated ion channels opening $[15,17]$. 


\section{Summary}

Both retraction and protrusions formation in cells are important in orchestrating complex biophysical phenomenon of cell electrotaxis [18]. Precise description of spatio-temporal dynamics of those processes is essential in understanding primary mechanisms responsible for detection of dcEF of physiological magnitude. This objective can be realized thanks to application of FRET biosensors described above, which are unique tools allowing to visualize activity of signaling hubs responsible for cellular processes with high spatial and temporal resolution.

This work is supported by grants from the National Science Centre, Poland (NCN) 2012/07/B/NZ3/02909 and the Polish Ministry of Science and Higher Education 7150/E-338/M/2017.

\section{REFERENCES}

[1] P. Devreotes, A.R. Horwitz, Cold Spring Harb. Perspect. Biol. 7, a005959 (2015).

[2] B. Alberts et al., Molecular Biology of the Cell, $4^{\text {th }}$ ed., Garland Science, New York, NY, 2002.

[3] S.J. Heasman, A.J. Ridley, Nat. Rev. Mol. Cell Biol. 9, 690 (2008).

[4] A. Schaefer, N.R. Reinhard, P.L. Hordijk, Small GTPases 5, e968004 (2014).

[5] M. Amano, M. Nakayama, K. Kaibuchi, Cytoskeleton 67, 545 (2010).

[6] D. Spiering et al., Methods Cell Biol. 114, 593 (2013).

[7] T. Förster, Ann. Phys. 437, 55 (1948).

[8] T. Förster, Zeitschrift Fur Naturforsch. Sect. A 4, 321 (1949).

[9] R.E. Dale, J. Eisinger, Biopolymers 13, 1573 (1974).

[10] J.R. Lakowicz, Principles of Fluorescence Spectroscopy, 2006, DOI:10.1007/978-1-4757-3061-6.

[11] M. Merkx, M.V. Golynskiy, L.H. Lindenburg, J.L. Vinkenborg, Biochem. Soc. Trans. 41, 1201 (2013).

[12] O. Pertz, L. Hodgson, R.L. Klemke, K.M. Hahn, Nature 440, 1069 (2006).

[13] Y. Moshfegh et al., Nat. Cell Biol. 16, 574 (2014).

[14] C.D. McCaig, A.M. Rajnicek, B. Song, M. Zhao, Physiol. Rev. 85, 943 (2005).

[15] B. Cortese, I.E. Palamà, S. D’Amone, G. Gigli, Integr. Biol. 6, 817 (2014).

[16] M.E. Mycielska, M.B.A. Djamgoz, J. Cell Sci. 117, 1631 (2004).

[17] G.M. Allen, A. Mogilner, J.A. Theriot, Curr. Biol. 23, 560 (2013).

[18] Y. Sun et al., Curr. Biol. 23, 569 (2013). 